IOS Press

\title{
Foreword by the Conference Chairs
}

Dear colleagues,

It is a great pleasure to present the proceedings of the 15th International Conference of the Association for the Advancement of Assistive Technology in Europe for your appreciation. The conference, taking place in Bologna (Italy) at the end of August 2019, covers as usual a wide variety of themes having to do with the relation between Technology and Disability. Beside the more traditional areas of Assistive Technology this includes Accessibility, Universal Design, Ambient Assisted Living, Smart environments and Internet of Things, Robotics, Technologies for independent living and self-management, Rehabilitation technology, Technology for Ageing, Augmentative and Alternative Communication, etc. It is a precise choice of the conference organisers, fully in line with the mission and positioning of AAATE as a European AT association of reference, not to specialise in any of those fields, but to keep this broad perspective, adopting the standpoint of those searching for effective assistive technology based solutions for their empowerment and for whom all solutions are open, as long as they work well. This approach makes the AAATE conference probably the most all-round AT conference in Europe and a biannual "must" for professionals supporting persons with disabilities and older adults. As a matter of fact, reading through the selected abstracts brought together in these Proceedings, the reader get a good picture of the broadness of the field and the wealth of competence represented by the authors.
We would like to thank the Programme Committee, in particular Lorenzo Desideri of AIAS Bologna, and the Scientific Committee Members for the work done in collecting, reviewing and selecting the abstracts here presented. They form the main scientific heritage of this conference, together with other reports that will be made from the educational sessions, the policy sessions and the product and prototype presentations. For organisational reasons it was not possible to include all that material in these proceedings.

Finally we would like to thank the authors that have chosen the AAATE conference to present their work. We have appreciated your effort and congratulate you with the high level of the work presented.

The Conference Chairs

Evert-Jan Hoogerwerf (AIAS Bologna onlus) Rabih Chattat (Alma Mater Studiorum University of

Bologna)

Luc de Witte (AAATE) 